\title{
Recombinant BCG-Prime and DNA-Boost Immunization Confers Mice with Enhanced Protection against Mycobacterium kansasii
}

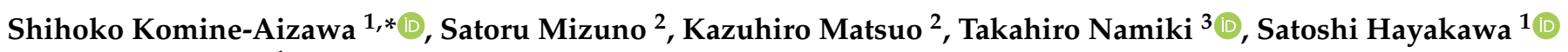 \\ and Mitsuo Honda ${ }^{1, *}$ \\ 1 Department of Pathology and Microbiology, Division of Microbiology, Nihon University School of Medicine, \\ Tokyo 173-8610, Japan; hayakawa.satoshi@nihon-u.ac.jp \\ 2 Japan BCG Laboratory, Tokyo 204-0022, Japan; s-mizuno@bcg.gr.jp (S.M.); matsuo@bcg.gr.jp (K.M.) \\ 3 Nihon University School of Medicine, Tokyo 173-8610, Japan; meta16076@g.nihon-u.ac.jp \\ * Correspondence: aizawa.shihoko@nihon-u.ac.jp (S.K.-A.); mitsuo77777@gmail.com (M.H.)
}

check for

updates

Citation: Komine-Aizawa, S.; Mizuno, S.; Matsuo, K.; Namiki, T.; Hayakawa, S.; Honda, M.

Recombinant BCG-Prime and DNA-Boost Immunization Confers Mice with Enhanced Protection against Mycobacterium kansasii. Vaccines 2021, 9, 1260. https:// doi.org/10.3390/vaccines9111260

Academic Editor: Ralph A. Tripp

Received: 11 October 2021

Accepted: 27 October 2021

Published: 1 November 2021

Publisher's Note: MDPI stays neutral with regard to jurisdictional claims in published maps and institutional affiliations.

Copyright: (c) 2021 by the authors. Licensee MDPI, Basel, Switzerland. This article is an open access article distributed under the terms and conditions of the Creative Commons Attribution (CC BY) license (https:/ / creativecommons.org/licenses/by/ $4.0 /)$.

\begin{abstract}
The incidence of infections with nontuberculous mycobacteria (NTM) has been increasing worldwide. The emergence of multidrug-resistant NTM is a serious clinical concern, and a vaccine for NTM has not yet been developed. We previously developed a new recombinant Bacillus Calmette-Guérin (rBCG) vaccine encoding the antigen 85B (Ag85B) protein of Mycobacterium kansasii-termed rBCG-Mkan85B-which was used together with a booster immunization with plasmid DNA expressing the same M. kansasii Ag85B gene (DNA-Mkan85B). We reported that rBCG-Mkan85B/DNA-Mkan85B prime-boost immunization elicited various NTM strain-specific $\mathrm{CD}^{+}$and $\mathrm{CD}^{+} \mathrm{T}$ cells and induced Mycobacterium tuberculosis-specific immunity. In this study, to investigate the protective effect against $M$. kansasii infection, we challenged mice vaccinated with a rBCG-Mkan85B or rBCG-Mkan85B/DNA-Mkan85B prime-boost strategy with virulent $M$. kansasii. Although BCG and rBCG-Mkan85B immunization each suppressed the growth of $M$. kansasii in the mouse lungs, the rBCG-Mkan85B/DNA-Mkan85B prime-boost vaccination reduced the bacterial burden more significantly. Moreover, the rBCG-Mkan85B/DNA-Mkan85B prime-boost vaccination induced antigen-specific $\mathrm{CD}^{+}$and $\mathrm{CD}^{+} \mathrm{T}$ cells. Our data suggest that rBCG-Mkan85B/DNAMkan85B prime-boost vaccination effectively enhances antigen-specific T cells. Our novel rBCG could be a potential alternative to clinical BCG for preventing various NTM infections.
\end{abstract}

Keywords: Mycobacterium kansasii; $\mathrm{CD}^{+} \mathrm{T}$ Cells; CD4 ${ }^{+}$T Cells; recombinant BCG

\section{Introduction}

Nontuberculous mycobacteria (NTM) are mycobacterium species other than the $M y$ cobacterium tuberculosis complex and Mycobacterium leprae, such as Mycobacterium avium, Mycobacterium intracellulare, and Mycobacterium kansasii. Recently, the incidence of NTM infection has been increasing worldwide [1-3]. Although NTM are considered not to cause airborne infection, NTM can cause pulmonary and extrapulmonary diseases in immunocompromised and even nonimmunocompromised individuals. Moreover, NTM infections have been reported to be resistant to treatment [4-8]. Recently, novel therapeutics against NTM have been developed [7,8], but effective prevention by vaccination is so far unknown.

Since NTM pathogens are intracellular, cell-mediated immunity plays a major role in protecting against NTM. Previous reports have indicated that protective immunity against tuberculosis (TB) and NTM infection might overlap [9]. Epidemiological data suggest that latent TB infection decreases the risk of NTM diseases [9], and a systematic review found that an increased proportion of NTM diseases were related to coincidental decreases in TB [10]. In addition, several reports have indicated that Bacillus Calmette-Guérin (BCG)which is the only available vaccine for TB — can induce protective immunity against NTM infection $[11,12]$. 
Previously, we developed a new recombinant BCG (rBCG) vaccine encoding the antigen 85B (Ag85B) protein of $M$. kansasii-termed rBCG-Mkan85B - which we used with a booster vaccination with plasmid DNA expressing the same M. kansasii Ag85B gene (DNA-Mkan85B) [13]. The Ag85 complex, which consists of three abundantly secreted proteins (Ag85A, Ag85B, and Ag85C), exhibits cell wall mycolyltransferase activity and plays an essential role in mycobacterial pathogenesis [14]. The Ag85 proteins have been reported to induce a strong cellular immune response against mycobacterium species such as M. tuberculosis and M. leprae $[15,16]$. We reported that rBCG-Mkan85B expressed Ag85B at levels 9.3-fold higher than parental BCG [13]. We also showed that the rBCGMkan85B/DNA-Mkan85B prime-boost scheme could elicit antigen-specific polyfunctional $\mathrm{CD}^{+} \mathrm{T}$ cells and $\mathrm{CD} 8^{+} \mathrm{T}$ cells [13]. Moreover, we identified two MHC-I $\left(\mathrm{H} 2-\mathrm{K}^{\mathrm{d}}\right)$-restricted epitopes that induced cross-reactive responses to $M$. tuberculosis and other related mycobacteria, including $M$. kansasii, in both BALB/c $\left(\mathrm{H}_{2}{ }^{\mathrm{d}}\right)$ mice and CB6F1 $\left(\mathrm{H}_{2} \mathrm{~b} / \mathrm{d}\right)$ mice [13]. The $\mathrm{H} 2-\mathrm{K}^{\mathrm{d}}$-restricted peptide epitopes elicited polyfunctional $\mathrm{CD} 8^{+} \mathrm{T}$ cell responses that were also highly cross-reactive with those of other proteins in the Ag85 complex [13]. Therefore, to confirm the protective effect of rBCG-Mkan85B/DNA-Mkan85B prime-boost vaccination against $M$. kansasii, we challenged rBCG-Mkan85B/DNA-Mkan85B-primeboost-vaccinated CB6F1 mice with $M$. kansasii and evaluated the protective effect and cellular immunity in this study.

\section{Materials and Methods}

\subsection{Animals}

Specific pathogen-free female CB6F1 mice, aged between 6 and 8 weeks, were purchased from Japan SLC Inc. (Shizuoka, Japan). All animal studies were carried out under institutional guidelines approved by the Nihon University Animal Care and Use Committee (AP19MED029-3), the institutional committee for gene recombination experiments (2018MED21), and biorisk management and control (30-10-4). The institutional animal experimental guidelines are in accordance with the ILAR Guide. Mice were allowed free access to sterile water and standard mouse food, and their physiological conditions were assessed every few days.

\subsection{BCG and rBCG-Mkan85B Culture}

We used the previously prepared rBCG-Mkan85B strain and DNA-Mkan85B plasmid [13] in this study.

BCG was cultured in Middlebrook 7H9 broth (Difco) supplemented with albumin dextrose complex (ADC) enrichment (Difco) and $0.05 \%$ Tween 80 at $37^{\circ} \mathrm{C}$. rBCG-Mkan85B was cultured in Middlebrook 7H9 broth (Difco) supplemented with ADC enrichment (Difco), $0.05 \%$ Tween 80 , and $10 \mathrm{mg} / \mathrm{mL}$ kanamycin at $37^{\circ} \mathrm{C}$. Bacterial culture density was monitored by measuring the absorbance at $470 \mathrm{~nm}$ and $600 \mathrm{~nm}$.

\subsection{Immunization}

Mice were immunized with the BCG vaccine or rBCG-Mkan85B at a concentration of $4 \times 10^{6} \mathrm{CFU}$ or $0.1 \mathrm{mg}$ of bacilli intradermally (i.d.), and $100 \mu \mathrm{g}$ of plasmid DNA in saline intramuscularly (i.m.) three times [17] (Figure S1A,B). We used three to four mice per group.

\subsection{Bacterial Infection}

M. kansasii Hauduroy 1955 was obtained from the RIKEN Bio Resource Center under a material transfer agreement (MTA). The bacteriological experiment was carried out under institutional guidelines approved by Nihon University biorisk management and control (3010-4). M. kansasii was grown in Middlebrook 7H9 broth (Difco) supplemented with ADC enrichment (Difco) with $0.05 \%$ Tween 80 at $37^{\circ} \mathrm{C}$ and harvested during the exponential growth stage. Infection of mice with $M$. kansasii was conducted intratracheally. Mice 
were anaesthetized with isoflurane and inoculated with $50 \mu \mathrm{L}$ of a bacterial suspension containing 1 to $10 \times 10^{4} \mathrm{CFU}$ of $M$. kansasii using a yellow pipet tip.

\subsection{Infection Assay}

At 6 weeks after infection, the mice were sacrificed, and the numbers of viable bacilli in their lungs were estimated. Lung tissues were homogenized with sterilized water. Tenfold serial dilutions of homogenate were inoculated onto duplicate 7H10-OADC agar plates (Difco) to determine bacterial loads. Colonies were counted after incubation for 3 weeks at $37^{\circ} \mathrm{C}$.

\subsection{Histopathology}

Fractioned lung samples from each mouse were fixed with 10\% neutral-buffered formalin and embedded in paraffin wax. The sections from these tissues were $4 \mathrm{~mm}$ thick and stained with hematoxylin and eosin (H\&E) or with the Ziehl-Neelsen stain for acid-fast bacilli.

\subsection{Polychromatic Flow Cytometry for Intracellular Cytokine Production}

Splenocytes and immune cells isolated from the lung were stimulated with a 9-mer Ag85B CD8 epitope peptide (YYQSGLSIV) (Pep8) [13], 15-mer Ag85B CD4 ${ }^{+}$epitope peptide (peptide-25: FQDAYNAAGGHNAVF) [18], or tuberculin PPD (PPD; $2 \mu \mathrm{g} / \mathrm{mL}$ ) and stained for cell-surface or intracellular cytokines such as IFN- $\gamma$ IL-2 and TNF, as described previously [13,17]. PPD was kindly supplied by the Japan BCG Laboratory. The Pep8 and peptide 25 were modified to match the amino acid sequence of $M$. kansasii. A seven-color flow cytometry panel was used to simultaneously analyze multiple cytokines at the singlecell level. The gating strategy used to identify cytokine-producing $\mathrm{CD}^{+}$and $\mathrm{CD}^{+} \mathrm{T}$ cells in mouse splenocytes is shown in Figure S2. Following in vitro stimulation for $6 \mathrm{~h}$, cells were incubated with LIVE/DEAD Fixable Dead Cell Stains (Thermo Fisher Scientific) to identify dead cells, followed by surface staining with the antibodies APC-conjugated anti-CD3, PerCP-Cy5.5-conjugated anti-CD8 (BioLegend), and PE-Cy7-conjugated anti-CD4 (BD). The cells were then fixed and permeabilized using BD Cytofix/Cytoperm (BD) and stained for IFN- $\gamma$ (PE), IL-2 (APC-Cy7), and TNF (Alexa Fluor 488) (BioLegend). Polyfunctional cells were defined as those making two or more cytokines using Boolean combinations [13]. FACS analysis was performed using a FACSVerse (BD) with FlowJo (BD) [13,17].

\subsection{Data Analysis and Statistics}

All comparisons among recombinant and control groups and among immunization groups were conducted using one-way ANOVA tests with the Tukey-Kramer test using the JMP program (SAS Institute) and R version 4.0.3 (R core team 2020). Data are expressed as the mean $\pm S D$.

\section{Results}

\subsection{BCG or rBCG-Mkan85B Vaccination Protects against M. kansasii Infection}

According to previous epidemiological reports, BCG vaccination can reduce the risk of NTM infection in humans [19-21]. In a mouse model, BCG vaccination was reported to induce cross-protective immunity against $M$. avium and $M$. abscessus [11]. Therefore, we first examined the protective efficacy of BCG and rBCG-Mkan85B against $M$. kansasii infection.

The CB6F1 (H2b/d) mice were either left unimmunized or immunized with BCG or rBCG-Mkan85B for 6 weeks, followed by a nasal exposure to a virulent $M$. kansasii strain for infection for another 6 weeks. The immunization schedule is shown in Figure S1A. Unimmunized mice produced $4.5 \times 10^{4}\left( \pm 1.5 \times 10^{4}\right)$ pulmonary CFU per animal. Although BCG and rBCG-Mkan85B vaccination both reduced the pulmonary CFU $\left(3.3 \times 10^{3} \pm 0.9 \times 10^{3}\right.$, and $5.0 \times 10^{3} \pm 1.4 \times 10^{3}$, respectively) $(p<0.01)$ (Figure 1), the efficacy of rBCG-Mkan85B was comparable to that of BCG. In the lungs of the unvaccinated mice, large granuloma 
nodules were predominant and consisted of epithelioid cells (Figure 2A,D). Acid-fast bacilli were detected in the granulomas using Ziehl-Neelsen staining (Figure 2G,J). Although granuloma nodules were also observed in the lungs of the BCG- or rBCG-Mkan85B-vaccinated animals (Figure $2 B, C$ ), the vaccination with BCG or rBCG-Mkan85B reduced granuloma nodule formation within the lungs, and few acid-fast bacilli were detected in the granulomas using Ziehl-Neelsen staining (Figure 2H,I).

\section{2. rBCG-Mkan85B/DNA-Mkan85B Prime-Boost Immunization Elicits Protection against Intratracheal Infection with $M$. kansasii}

Next, we evaluated the efficacy of the rBCG-Mkan85B/DNA-Mkan85B prime-boost vaccination against $M$. kansasii infection. The CB6F1 mice were either left unimmunized or immunized with BCG or rBCG-Mkan85B/DNA-Mkan85B and then challenged with a virulent $M$. kansasii strain for another 6 weeks. The immunization schedule is shown in Figure S1B. As shown in Figure 3, the rBCG-Mkan85B/DNA-Mkan85B vaccination drastically reduced the bacterial burden (Figure 3). Live bacteria were not detected in the lungs of the rBCG-Mkan85B/DNA-Mkan85B-vaccinated CB6F1 mice except for one mouse (Figure 3).

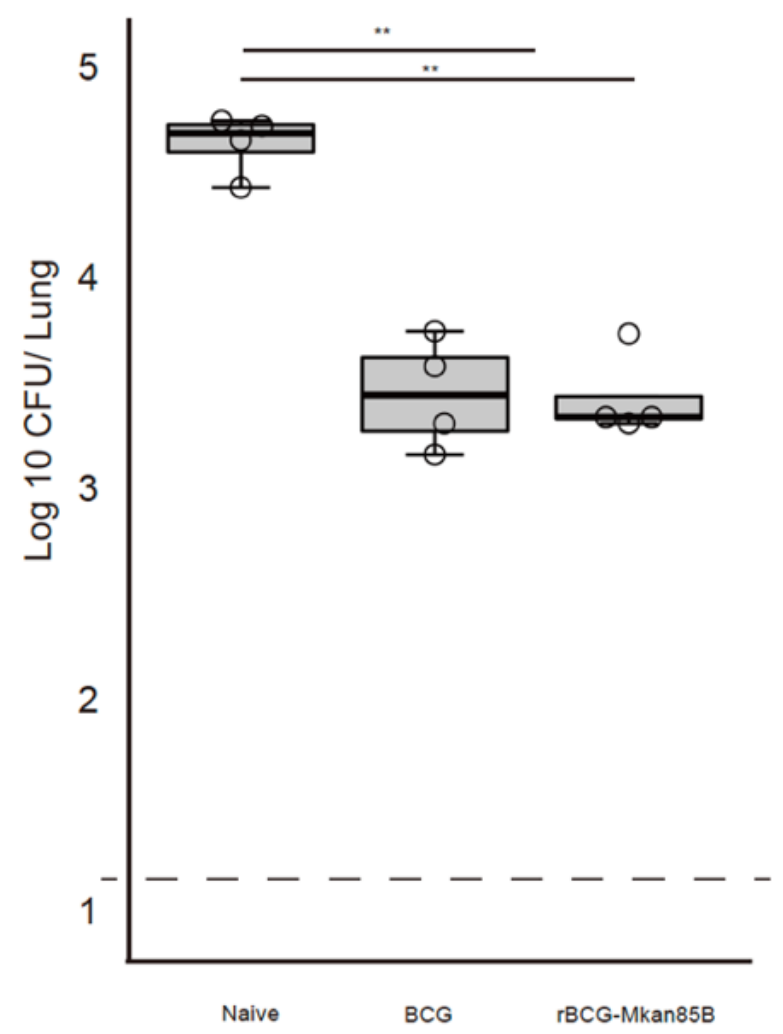

Figure 1. BCG or rBCG-Mkan85B vaccination protects against $M$. kansasii infection. The CB6F1 $(\mathrm{H} 2 \mathrm{~b} / \mathrm{d})$ mice were either left unimmunized or immunized with BCG or rBCG-Mkan85B for 6 weeks, followed by intratracheal infection with a virulent $M$. kansasii strain. The mice were sacrificed at 6 weeks after M. kansasii infection. The immunization schedule is shown Figure S1A. The number of M. kansasii in individual mouse lungs was analyzed using the colony assay method. The data represent two independent experiments with three to four mice per group. The detection limit for bacilli in the tissue homogenate was $15 \mathrm{CFU}$. The error bars represent the SD. ${ }^{* *} p<0.01$. 

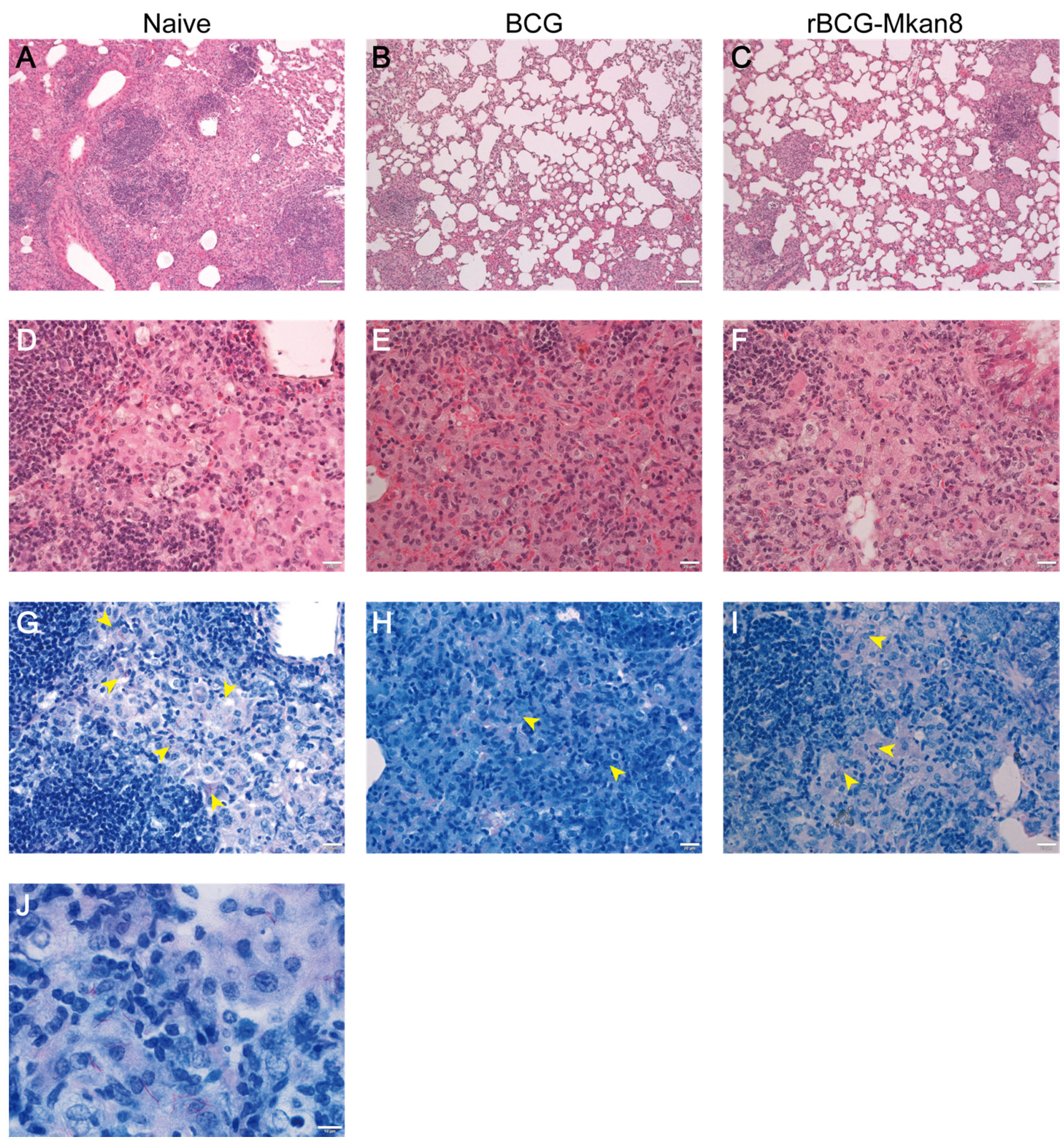

Figure 2. Histopathology of lungs from $M$. kansasii-infected mice. Representative pathological observations of the lungs of the unvaccinated (A,D,G,J), BCG-vaccinated (B,E,H), and rBCG-Mkan85B-vaccinated (C,F,I) mice 6 weeks after M. kansasii infection are shown. First-row panels, $100 \times$ of HE-stained specimens; second-row panels, $400 \times$ of HE-stained specimens; third-row panels, $400 \times$ of Ziehl-Neelsen-stained specimens; (panel J), 1000 $\times$ of Ziehl-Neelsen-stained specimens. The yellow arrowheads indicate the acid-fast bacilli. Bars: $100 \mu \mathrm{m}$ (first-row panels), $20 \mu \mathrm{m}$ (second- and third-row panels), and $10 \mu \mathrm{m}$ (panel J). 


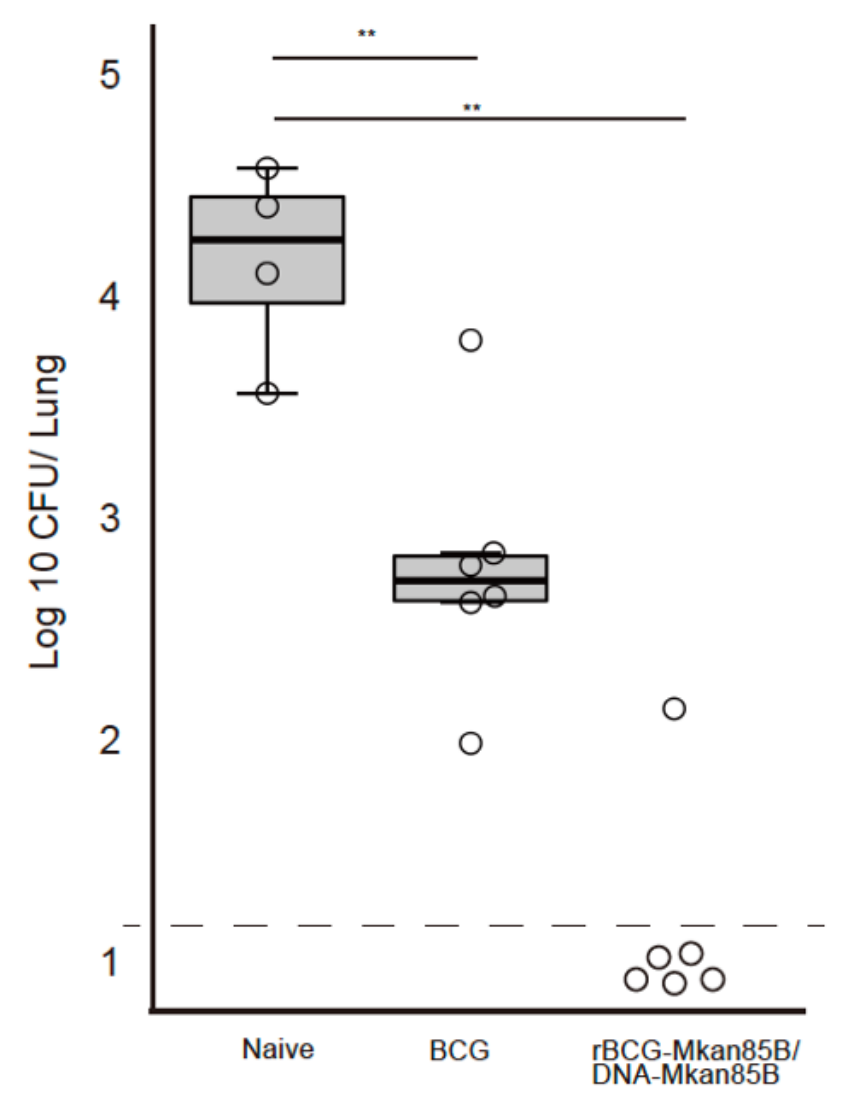

Figure 3. rBCG-Mkan85B/DNA-Mkan85B vaccination protects against $\mathrm{M}$. kansasii infection. The CB6F1 mice were either left unimmunized or immunized with BCG or rBCG-Mkan85B/DNAMkan85B and then challenged with a virulent M. kansasii strain for another 6 weeks. The immunization schedule is shown in Figure S1B. The mice were sacrificed at 6 weeks after M. kansasii infection. The number of M. kansasii in individual mouse lungs was analyzed using the colony assay method. The data represent two independent experiments with five to six mice per group. The detection limit for bacilli in the tissue homogenate was $15 \mathrm{CFU}$. The error bars represent the SD. ${ }^{* *} p<0.01$.

3.3. rBCG-Mkan85B/DNA-Mkan85B Prime-Boost Vaccination Induced Polyfunctional CD8 ${ }^{+} T$ Cells Specific for the Major Secretory Protein Ag85B in the Lung and Spleen

To analyze the adaptive immune responses induced by BCG, rBCG-Mkan85B, and rBCG-Mkan85B/DNA-Mkan85B vaccination, we examined the induction of antigenspecific polyfunctional T cells using an intracellular cytokine staining (ICS) method.

Splenocytes were obtained from each mouse vaccinated with BCG or rBCG-Mkan85B at six weeks after M. kansasii infection (Figure S1A). The cells were stimulated with 9-mer Ag85B CD8 epitope peptides (Pep8) [13], the 15-mer Ag85B CD4 epitope peptide (peptide 25) [18], or PPD (2 $\mu \mathrm{g} / \mathrm{mL})$ in vitro. The Pep 8 and peptide 25 were modified to match the amino acid sequence of $M$. kansasii. Polyfunctional T cells, defined as those that produced two or three cytokines, were analyzed using flow cytometry. The BCG and rBCG-Mkan85B vaccination elicited similar levels of PPD-specific polyfunctional CD4 ${ }^{+}$T cells (Figure $4 \mathrm{~A}, \mathrm{~B}$ ). The rBCG-Mkan85B vaccination induced higher levels of peptide 25-specific polyfunctional $\mathrm{CD}^{+} \mathrm{T}$ cells than the BCG vaccination, but the difference was not significant (Figure 4A,B). On the other hand, the BCG or rBCG-Mkan85B vaccination alone could not induce $M$. kansasii-specific CD8 ${ }^{+} \mathrm{T}$ cells (Figure $4 \mathrm{~A}, \mathrm{~B}$ ). These results were consistent with our previous reports showing that $B C G$ immunization could not induce polyfunctional $\mathrm{CD}^{+} \mathrm{T}$ cells specific to M. tuberculosis Ag85B [13]. 


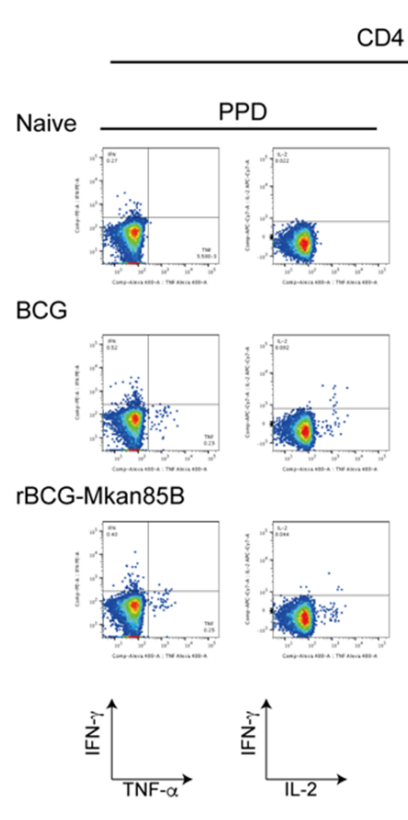

PPD
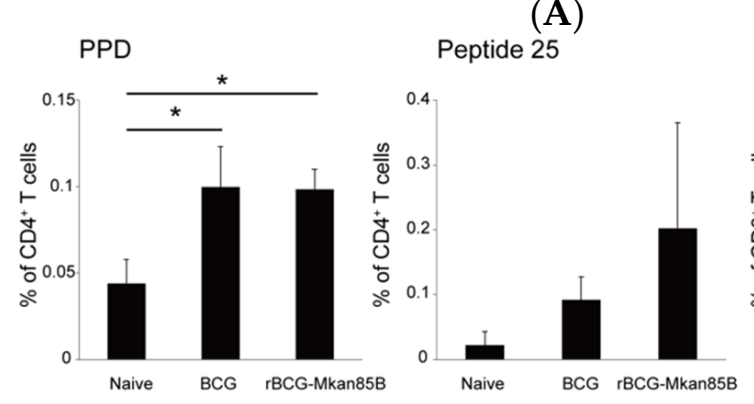

(B)
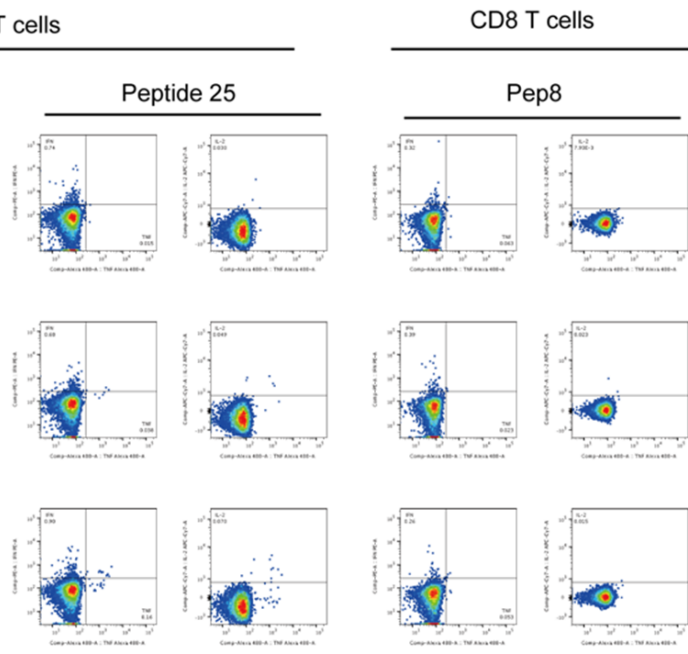

(A)

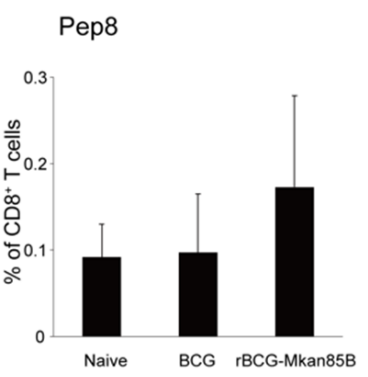

Figure 4. M. kansasii-specific polyfunctional $\mathrm{CD}^{+}$and $\mathrm{CD} 8^{+} \mathrm{T}$ cells in BCG- or rBCG-Mkan85Bvaccinated CB6F1 mice. (A) A representative fluorogram of epitope-specific polyfunctional CD4 ${ }^{+}$ and $\mathrm{CD}^{+} \mathrm{T}$ cell inductions in splenocytes from unvaccinated and vaccinated mice infected with M. kansasii; TNF is shown on the $x$-axis and IFN- $\gamma$ is shown on the $y$-axis. (B) Polyfunctional $\mathrm{CD}^{+}$and $\mathrm{CD}^{+}{ }^{+} \mathrm{T}$ cells from unvaccinated and vaccinated CB6F1 mice infected with $M$. kansasii. Polyfunctional $\mathrm{CD}^{+} \mathrm{T}$ cell induction by stimulation with PPD (left panel), polyfunctional $\mathrm{CD} 4^{+} \mathrm{T}$ cell induction by stimulation with peptide 25 (middle panel), and polyfunctional $\mathrm{CD}^{+} \mathrm{T}$ cell induction by stimulation with Pep8 (right panel) in unvaccinated, BCG-, and rBCG-Mkan85B-vaccinated mice are shown. The data represent two independent experiments with three to four mice per group. The error bars represent the SD. ${ }^{*} p<0.05$.

Next, we investigated the immune responses induced by rBCG-Mkan85B/DNAMkan85B prime-boost vaccination using the same method. In addition to splenocytes, immune cells isolated from the lungs were also analyzed. As shown in Figure 5, the rBCGMkan85B/DNA-Mkan85B immunization induced both $M$. kansasii-specific polyfunctional $\mathrm{CD}^{+}$and $\mathrm{CD} 8^{+} \mathrm{T}$ cells (Figure 5). Interestingly, M. kansasii-specific polyfunctional CD4 ${ }^{+}$ and $\mathrm{CD} 8^{+} \mathrm{T}$ cells were more strongly induced in the lung than in the spleen by the rBCGMkan85B immunization followed by the DNA-Mkan85B boost (Figure 5A,B). 


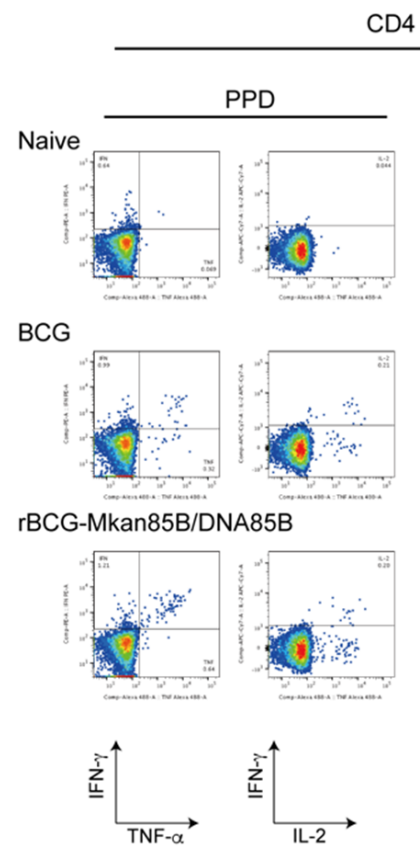

Spleen
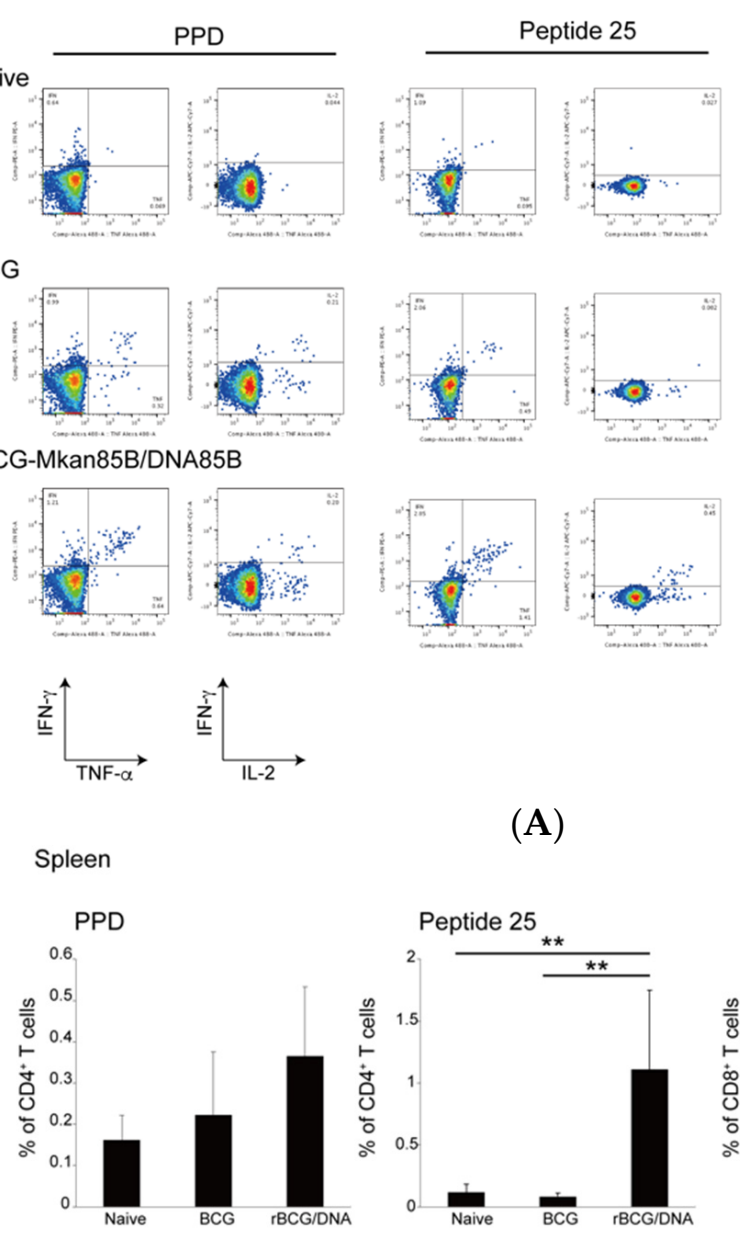

(A)
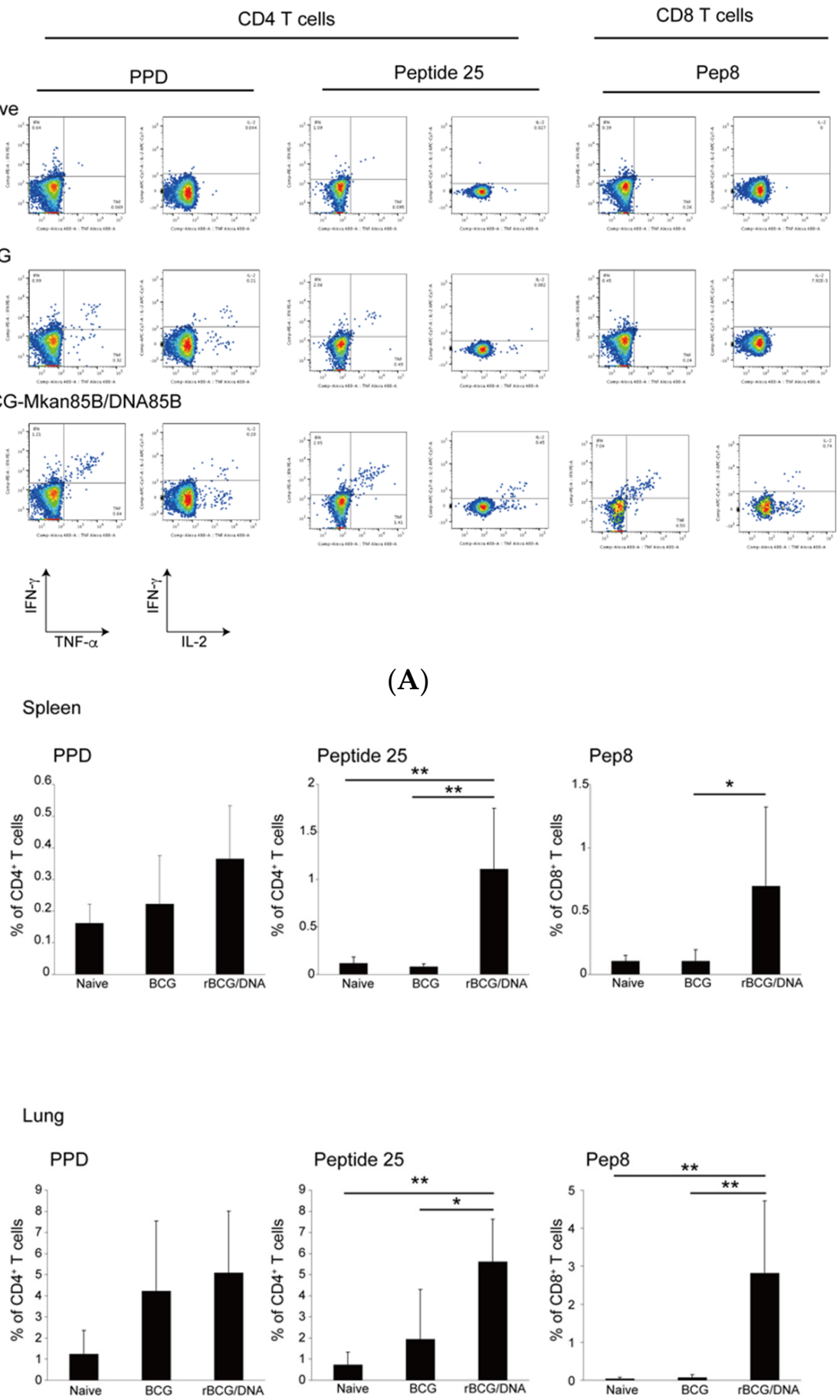

(B)

Figure 5. M. kansasii-specific polyfunctional $\mathrm{CD}^{+}$and $\mathrm{CD}^{+} \mathrm{T}$ cells in rBCG-Mkan85B/DNAMkan85B-prime-boost-vaccinated CB6F1 mice. (A) A representative fluorogram of epitope-specific polyfunctional $\mathrm{CD}^{+}$and $\mathrm{CD} 8^{+} \mathrm{T}$ cell inductions in splenocytes from unvaccinated and vaccinated CB6F1 mice infected with M. kansasii; TNF is shown on the $x$-axis and IFN- $\gamma$ is shown on the $y$-axis. (B) Polyfunctional $\mathrm{CD} 4^{+}$and $\mathrm{CD}^{+} \mathrm{T}$ cells from the unvaccinated and vaccinated $\mathrm{CB} 6 \mathrm{~F} 1$ mice infected with M. kansasii. Polyfunctional $\mathrm{CD}^{+} \mathrm{T}$ cell induction by stimulation with PPD (left panel), polyfunctional CD4+ T cell induction by stimulation with peptide 25 (middle panel), and polyfunctional $\mathrm{CD} 8^{+} \mathrm{T}$ cell induction by stimulation with Pep8 (right panel) in unvaccinated, BCG-, and rBCG-Mkan85B/DNA-Mkan85B-vaccinated mice are shown. The data represent two independent experiments with five to six mice per group. The error bars represent the SD. ${ }^{*} p<0.05$. ** $p<0.01$. 


\section{Discussion}

Cellular immunity plays an important role in controlling infections by intracellular pathogens, such as M. tuberculosis, NTM, and viruses. Previous studies by us and other researchers have shown that BCG immunization only weakly activates $C D 8^{+} \mathrm{T}$ cells, in contrast to its robust activation of $\mathrm{CD} 4^{+} \mathrm{T}$ cells $[13,22,23]$. We previously suggested that rBCG-prime and DNA-boost strategies could induce CD8 ${ }^{+} \mathrm{T}$ cell immunity to Ag85B of the $M$. tuberculosis [13] and HIV envelope proteins [17,24]. In this study, we observed that the rBCG-Mkan85B/DNA-Mkan85B prime-boost scheme induced antigen-specific polyfunctional $\mathrm{CD} 8^{+} \mathrm{T}$ cells, which produced double or triple combinations of IFN- $\gamma$, IL-2, and TNF in addition to polyfunctional $\mathrm{CD} 4^{+} \mathrm{T}$ cells.

Generally, NTM patients are considered to have been infected by environmental NTM, not by NTM from other patients. In other words, NTM do not cause airborne or droplet infections, unlike TB and SARS-CoV-2, the latter of which is responsible for coronavirus disease 2019 (COVID-19). However, increases in NTM incidence rates have been reported recently [1,2]. Moreover, the emergence of multidrug-resistant NTM is a serious clinical concern, and vaccines for NTM are not available currently. BCG has been used as a TB vaccine, and some researchers have reported that BCG vaccination can reduce the risk of NTM infection, such as infection with $M$. avium-intracellulare complex (MAC) [19-21], $M$. malmoense, M. marinum, or M. kansasii [20]. Abate et al. reported that BCG vaccination induced M. avium and M. abscessus cross-reactive T cells in humans [11]. Previously, we reported that various $\mathrm{CD} 8^{+} \mathrm{T}$ cell epitopes of the mycobacterial Ag85 complex elicited similar levels of cytokine production by polyfunctional $\mathrm{T}$ cells in mice vaccinated with an rBCG-Mkan85B/DNA-Mkan85B prime-boost strategy [13].

In the present study, we first challenged unvaccinated and BCG- or rBCG-Mkan85Bvaccinated mice with virulent $M$. kansasii. We showed that both BCG and rBCG-Mkan85B could protect the mice from virulent $M$. kansasii infection. However, contrary to our expectation, the efficacy of rBCG-Mkan85B vaccination was not superior to that of BCG vaccination. An ICS analysis revealed that $M$. kansasii antigen-specific polyfunctional $\mathrm{CD}^{+}$ $\mathrm{T}$ cells were equally induced by BCG and $\mathrm{rBCG}-\mathrm{Mkan} 85 \mathrm{~B}$ vaccination. However, similar to previous reports $[13,22,23,25]$, we observed that $B C G$ immunization and in vitro restimulation with $M$. kansasii peptides could not induce antigen-specific polyfunctional CD8 ${ }^{+} \mathrm{T}$ cells. Moreover, the present study demonstrated that rBCG-Mkan85B vaccination alone was not sufficient to induce M. kansasii Ag85B-specific polyfunctional CD8 ${ }^{+} \mathrm{T}$ cells. Therefore, we immunized mice with rBCG-Mkan85B and boosted them with DNA-Mkan85B three times. BCG and recombinant BCG prime-boost immunization were reported to be beneficial strategies for inducing antigen-specific $\mathrm{CD}^{+} \mathrm{T}$ cells $[13,17,24]$. In the present study, the bacterial burden in the lungs was remarkably reduced in the mice vaccinated with the rBCG-Mkan85B/DNA-Mkan85B prime-boost strategy. In addition, we revealed that rBCG-Mkan85B/DNA-Mkan85B vaccination could induce antigen-specific polyfunctional $\mathrm{CD}^{+}$and $\mathrm{CD} 4^{+} \mathrm{T}$ cells. Regarding antigen-specific $\mathrm{CD} 4^{+} \mathrm{T}$ cells, several critical roles for eliminating mycobacteria have been reported $[11,26,27]$. In fact, AIDS patients with low $\mathrm{CD}^{+} \mathrm{T}$ cell counts have a high risk of developing NTM infection. In addition to that of $\mathrm{CD}^{+} \mathrm{T}$ cells, the importance of $\mathrm{CD} 8^{+} \mathrm{T}$ cells to protecting against mycobacterial infection was revealed by previous studies by other researchers. For instance, the airborne infection of mice [28], calves [29], and rhesus macaques [27] with M. avium induced the activation of both $\mathrm{CD}^{+} \mathrm{T}$ cells and $\mathrm{CD} 8^{+} \mathrm{T}$ cells. However, the significance of $\mathrm{CD} 8^{+} \mathrm{T}$ cells in protection against NTM infection, especially M. avium infection, has been controversial. Although the depletion or genetic elimination of $\mathrm{CD}^{+} \mathrm{T}$ cells significantly decreases immunological responses to TB and increases susceptibility to $M$. tuberculosis $[30,31]$, the depletion of $\mathrm{CD}^{+} \mathrm{T}$ cells was reported to be unrelated to M. avium infection [32]. Gilbertson et al. suggested that the bystander activation of $\mathrm{CD}^{+} \mathrm{T}$ cells occurred during experimental $M$. avium infection [28]. On the other hand, the role of $\mathrm{CD} 8^{+} \mathrm{T}$ cells in $M$. kansasii infection has not been investigated thoroughly. In the present study, although BCG or rBCG-Mkan85B immunization could activate only $M$. kansasii-specific polyfunctional $\mathrm{CD}^{+} \mathrm{T}$ cells, $\mathrm{rBCG}-$ 
Mkan85B/DNA-Mkan85B prime-boost vaccination was able to elicit $M$. kansasii-specific polyfunctional $\mathrm{CD} 8^{+} \mathrm{T}$ cells. Nevertheless, the BCG- or rBCG-Mkan85B-immunized mice were shown to be protected against $M$. kansasii infection, and the rBCG-Mkan85B/DNAMkan85B prime-boost strategy drastically reduced the bacterial burden in the lungs of the mice. The results suggest a role for antigen-specific polyfunctional CD8 ${ }^{+} \mathrm{T}$ cells in protecting against $M$. kansasii infection.

Since the end of 2019, COVID-19 has been a serious public health concern worldwide. Looking back on history, human beings have suffered from many kinds of infections. We have to continue to fight various pathogenic microorganisms and infectious diseases. Vaccines are the gold standard for protecting against infectious diseases. One of the aims of a vaccine is to induce effective neutralizing antibodies against the target pathogen. To protect against several pathogenic microbes, such as mycobacteria, the activation of cellular immunity is required in addition to the induction of humoral immunity. BCG has been used globally as a live attenuated vaccine against TB for a century. Various recombinant BCG strains have been designed, developed, and evaluated as novel vaccines against various infectious diseases. For instance, several rBCG strains expressing HIV antigens, such as HIV gag and env, have been reported to induce anti-HIV neutralizing antibodies and antigen-specific $\mathrm{CD} 4^{+}$and $\mathrm{CD} 8^{+} \mathrm{T}$ cells $[24,33,34]$. We and others reported that a prime-boost strategy could elicit relatively high levels of immunological activity against pathogens [13,33,35-37]. Moreover, BCG has the advantages of providing safety to healthy subjects and potent adjuvant activities. Therefore, rBCG might be a candidate for use in novel vaccines against emerging infectious diseases for which vaccines have not yet been developed.

\section{Conclusions}

In conclusion, we demonstrated that BCG and rBCG-Mkan85B immunization protected $\mathrm{CB} 6 \mathrm{~F} 1$ mice from $M$. kansasii infection by inducing $M$. kansasii-specific polyfunctional $\mathrm{CD}^{+} \mathrm{T}$ cells. Furthermore, rBCG-Mkan85B/DNA-Mkan85B prime-boost vaccination induced antigen-specific $\mathrm{CD} 4^{+}$and $\mathrm{CD} 8^{+} \mathrm{T}$ cells and drastically reduced the $\mathrm{CFU}$ of $M$. kansasii in the lungs of vaccinated mice. Our data suggest that the combination of rBCG expressing Ag85B derived from M. kansasii with DNA that also expresses Ag85B derived from $M$. kansasii may be effective in enhancing antigen-specific $\mathrm{T}$ cells, resulting in more efficient control of $M$. kansasii infection.

Supplementary Materials: The following are available online at https:/ /www.mdpi.com/article/10 .3390 /vaccines9111260/s1, Figure S1: Immunization Schedule, Figure S2: Gating tree for functional characterization of distinct populations of responding $\mathrm{CD}^{+}$or $\mathrm{CD} 8^{+} \mathrm{T}$ cells using polychromatic flow cytometry.

Author Contributions: Conceptualization, S.K.-A. and M.H.; Data curation, S.K.-A.; Formal analysis, S.K.-A. and T.N.; Funding acquisition, S.K.-A.; Investigation, S.K.-A.; Methodology, M.H.; Project administration, S.K.-A.; Resources, S.M. and K.M.; Supervision, S.M., K.M., S.H. and M.H.; Writingoriginal draft, S.K.-A.; Writing-review \& editing, S.M., K.M., S.H. and M.H. All authors have read and agreed to the published version of the manuscript.

Funding: This research was funded by the JSPS KAKENHI, grant number 19K08939 to S.K.-A.

Institutional Review Board Statement: The study was conducted according to the guidelines of the Declaration of Helsinki, and approved by the Nihon University Animal Care and Use Committee (AP19MED029-3), the institutional committee for gene recombination experiments (2018MED21), and biorisk management and control (30-10-4). The institutional animal experimental guidelines are in accordance with the ILAR Guide.

Data Availability Statement: The authors declare that the data supporting the findings of this study are available within the paper. 
Acknowledgments: We thank Maruoka and Soda, Division of Respiratory Medicine, Nihon University School of Medicine, for their helpful advice for infection experiments. We also thank American Journal Experts for editing the English language in this manuscript.

Conflicts of Interest: The authors declare no conflict of interest.

\section{References}

1. Namkoong, H.; Kurashima, A.; Morimoto, K.; Hoshino, Y.; Hasegawa, N.; Ato, M.; Mitarai, S. Epidemiology of Pulmonary Nontuberculous Mycobacterial Disease, Japan. Emerg. Infect. Dis. 2016, 22, 1116-1117. [CrossRef]

2. Prevots, D.R.; Marras, T.K. Epidemiology of human pulmonary infection with nontuberculous mycobacteria: A review. Clin. Chest Med. 2015, 36, 13-34. [CrossRef]

3. Lee, Y.M.; Kim, M.J.; Kim, Y.J. Increasing Trend of Nontuberculous Mycobacteria Isolation in a Referral Clinical Laboratory in South Korea. Medicina 2021, 57, 720. [CrossRef] [PubMed]

4. Maekura, R.; Okuda, Y.; Hirotani, A.; Kitada, S.; Hiraga, T.; Yoshimura, K.; Yano, I.; Kobayashi, K.; Ito, M. Clinical and prognostic importance of serotyping Mycobacterium avium-Mycobacterium intracellulare complex isolates in human immunodeficiency virus-negative patients. J. Clin. Microbiol. 2005, 43, 3150-3158. [CrossRef]

5. de Mello, K.G.; Mello, F.C.; Borga, L.; Rolla, V.; Duarte, R.S.; Sampaio, E.P.; Holland, S.M.; Prevots, D.R.; Dalcolmo, M.P. Clinical and therapeutic features of pulmonary nontuberculous mycobacterial disease, Brazil, 1993-2011. Emerg. Infect. Dis. 2013, 19, 393-399. [CrossRef]

6. Griffith, D.E.; Aksamit, T.; Brown-Elliott, B.A.; Catanzaro, A.; Daley, C.; Gordin, F.; Holland, S.M.; Horsburgh, R.; Huitt, G.; Iademarco, M.F.; et al. An official ATS/IDSA statement: Diagnosis, treatment, and prevention of nontuberculous mycobacterial diseases. Am. J. Respir Crit. Care Med. 2007, 175, 367-416. [CrossRef] [PubMed]

7. Silwal, P.; Kim, I.S.; Jo, E.K. Autophagy and Host Defense in Nontuberculous Mycobacterial Infection. Front. Immunol. 2021, 12, 728742. [CrossRef] [PubMed]

8. Kasperbauer, S.H.; De Groote, M.A. The treatment of rapidly growing mycobacterial infections. Clin. Chest Med. 2015, 36, 67-78. [CrossRef]

9. Horsburgh, C.R., Jr.; Hanson, D.L.; Jones, J.L.; Thompson, S.E., 3rd. Protection from Mycobacterium avium complex disease in human immunodeficiency virus-infected persons with a history of tuberculosis. J. Infect. Dis. 1996, 174, 1212-1217. [CrossRef]

10. Brode, S.K.; Daley, C.L.; Marras, T.K. The epidemiologic relationship between tuberculosis and non-tuberculous mycobacterial disease: A systematic review. Int. J. Tuberc. Lung Dis. 2014, 18, 1370-1377. [CrossRef]

11. Abate, G.; Hamzabegovic, F.; Eickhoff, C.S.; Hoft, D.F. BCG Vaccination Induces M. avium and M. abscessus Cross-Protective Immunity. Front. Immunol. 2019, 10, 234. [CrossRef] [PubMed]

12. Zimmermann, P.; Finn, A.; Curtis, N. Does BCG Vaccination Protect Against Nontuberculous Mycobacterial Infection? A Systematic Review and Meta-Analysis. J. Infect. Dis. 2018, 218, 679-687. [CrossRef] [PubMed]

13. Komine-Aizawa, S.; Jiang, J.; Mizuno, S.; Hayakawa, S.; Matsuo, K.; Boyd, L.F.; Margulies, D.H.; Honda, M. MHC-restricted Ag85B-specific CD8(+) T cells are enhanced by recombinant BCG prime and DNA boost immunization in mice. Eur. J. Immunol. 2019, 49, 1399-1414. [CrossRef]

14. Wiker, H.G.; Harboe, M. The antigen 85 complex: A major secretion product of Mycobacterium tuberculosis. Microbiol. Rev. 1992, 56, 648-661. [CrossRef]

15. Launois, P.; Drowart, A.; Bourreau, E.; Couppie, P.; Farber, C.M.; Van Vooren, J.P.; Huygen, K. T cell reactivity against mycolyl transferase antigen 85 of M. tuberculosis in HIV-TB coinfected subjects and in AIDS patients suffering from tuberculosis and nontuberculous mycobacterial infections. Clin. Dev. Immunol. 2011, 2011, 640309. [CrossRef] [PubMed]

16. Launois, P.; DeLeys, R.; Niang, M.N.; Drowart, A.; Andrien, M.; Dierckx, P.; Cartel, J.L.; Sarthou, J.L.; Van Vooren, J.P.; Huygen, K. T-cell-epitope mapping of the major secreted mycobacterial antigen Ag85A in tuberculosis and leprosy. Infect. Immun. 1994, 62, 3679-3687. [CrossRef]

17. Honda, M.; Wang, R.; Kong, W.P.; Kanekiyo, M.; Akahata, W.; Xu, L.; Matsuo, K.; Natarajan, K.; Robinson, H.; Asher, T.E.; et al. Different vaccine vectors delivering the same antigen elicit CD8+ T cell responses with distinct clonotype and epitope specificity. J. Immunol. 2009, 183, 2425-2434. [CrossRef] [PubMed]

18. Kariyone, A.; Tamura, T.; Kano, H.; Iwakura, Y.; Takeda, K.; Akira, S.; Takatsu, K. Immunogenicity of Peptide-25 of Ag85B in Th1 development: Role of IFN-gamma. Int. Immunol. 2003, 15, 1183-1194. [CrossRef] [PubMed]

19. Katila, M.L.; Brander, E.; Backman, A. Neonatal BCG vaccination and mycobacterial cervical adenitis in childhood. Tubercle 1987, 68, 291-296. [CrossRef]

20. Romanus, V.; Hallander, H.O.; Wahlen, P.; Olinder-Nielsen, A.M.; Magnusson, P.H.; Juhlin, I. Atypical mycobacteria in extrapulmonary disease among children. Incidence in Sweden from 1969 to 1990, related to changing BCG-vaccination coverage. Tuber. Lung Dis. 1995, 76, 300-310. [CrossRef]

21. Trnka, L.; Dankova, D.; Svandova, E. Six years' experience with the discontinuation of BCG vaccination. 4. Protective effect of BCG vaccination against the Mycobacterium avium intracellulare complex. Tuber. Lung Dis. 1994, 75, 348-352. [CrossRef]

22. Ottenhoff, T.H.; Kaufmann, S.H. Vaccines against tuberculosis: Where are we and where do we need to go? PLoS Pathog. 2012, 8 , e1002607. [CrossRef] 
23. Ryan, A.A.; Nambiar, J.K.; Wozniak, T.M.; Roediger, B.; Shklovskaya, E.; Britton, W.J.; Fazekas de St Groth, B.; Triccas, J.A. Antigen load governs the differential priming of CD8 T cells in response to the bacille Calmette Guerin vaccine or Mycobacterium tuberculosis infection. J. Immunol. 2009, 182, 7172-7177. [CrossRef]

24. Honda, M.; Matsuo, K.; Nakasone, T.; Okamoto, Y.; Yoshizaki, H.; Kitamura, K.; Sugiura, W.; Watanabe, K.; Fukushima, Y.; Haga, S.; et al. Protective immune responses induced by secretion of a chimeric soluble protein from a recombinant Mycobacterium bovis bacillus Calmette-Guerin vector candidate vaccine for human immunodeficiency virus type 1 in small animals. Proc. Natl. Acad. Sci. USA 1995, 92, 10693-10697. [CrossRef]

25. Mazzaccaro, R.J.; Gedde, M.; Jensen, E.R.; van Santen, H.M.; Ploegh, H.L.; Rock, K.L.; Bloom, B.R. Major histocompatibility class I presentation of soluble antigen facilitated by Mycobacterium tuberculosis infection. Proc. Natl. Acad. Sci. USA 1996, 93, 11786-11791. [CrossRef]

26. Kwon, B.E.; Ahn, J.H.; Park, E.K.; Jeong, H.; Lee, H.J.; Jung, Y.J.; Shin, S.J.; Jeong, H.S.; Yoo, J.S.; Shin, E.; et al. B Cell-Based Vaccine Transduced With ESAT6-Expressing Vaccinia Virus and Presenting alpha-Galactosylceramide Is a Novel Vaccine Candidate against ESAT6-Expressing Mycobacterial Diseases. Front. Immunol. 2019, 10, 2542. [CrossRef]

27. Winthrop, K.; Rivera, A.; Engelmann, F.; Rose, S.; Lewis, A.; Ku, J.; Bermudez, L.; Messaoudi, I. A Rhesus Macaque Model of Pulmonary Nontuberculous Mycobacterial Disease. Am. J. Respir. Cell Mol. Biol. 2016, 54, 170-176. [CrossRef]

28. Gilbertson, B.; Germano, S.; Steele, P.; Turner, S.; Fazekas de St Groth, B.; Cheers, C. Bystander activation of CD8+ T lymphocytes during experimental mycobacterial infection. Infect. Immun. 2004, 72, 6884-6891. [CrossRef]

29. Stabel, J.R.; Waters, W.R.; Bannantine, J.P.; Palmer, M.V. Disparate host immunity to Mycobacterium avium subsp. paratuberculosis antigens in calves inoculated with M. avium subsp. paratuberculosis, M. avium subsp. avium, M. kansasii, and M. bovis. Clin. Vaccine Immunol. 2013, 20, 848-857. [CrossRef]

30. Chen, C.Y.; Huang, D.; Wang, R.C.; Shen, L.; Zeng, G.; Yao, S.; Shen, Y.; Halliday, L.; Fortman, J.; McAllister, M.; et al. A critical role for CD8 T cells in a nonhuman primate model of tuberculosis. PLoS Pathog. 2009, 5, e1000392. [CrossRef]

31. Woodworth, J.S.; Behar, S.M. Mycobacterium tuberculosis-specific CD8+ T cells and their role in immunity. Crit. Rev. Immunol. 2006, 26, 317-352. [CrossRef] [PubMed]

32. Bermudez, L.E.; Petrofsky, M. Host defense against Mycobacterium avium does not have an absolute requirement for major histocompatibility complex class I-restricted T cells. Infect. Immun. 1999, 67, 3108-3111. [CrossRef] [PubMed]

33. Chapman, R.; Bourn, W.R.; Shephard, E.; Stutz, H.; Douglass, N.; Mgwebi, T.; Meyers, A.; Chin'ombe, N.; Williamson, A.L. The use of directed evolution to create a stable and immunogenic recombinant BCG expressing a modified HIV-1 Gag antigen. PLoS ONE 2014, 9, e103314. [CrossRef]

34. Kanekiyo, M.; Matsuo, K.; Hamatake, M.; Hamano, T.; Ohsu, T.; Matsumoto, S.; Yamada, T.; Yamazaki, S.; Hasegawa, A.; Yamamoto, N.; et al. Mycobacterial codon optimization enhances antigen expression and virus-specific immune responses in recombinant Mycobacterium bovis bacille Calmette-Guerin expressing human immunodeficiency virus type $1 \mathrm{Gag}$. J. Virol. 2005, 79, 8716-8723. [CrossRef] [PubMed]

35. Ami, Y.; Izumi, Y.; Matsuo, K.; Someya, K.; Kanekiyo, M.; Horibata, S.; Yoshino, N.; Sakai, K.; Shinohara, K.; Matsumoto, S.; et al. Priming-boosting vaccination with recombinant Mycobacterium bovis bacillus Calmette-Guerin and a nonreplicating vaccinia virus recombinant leads to long-lasting and effective immunity. J. Virol. 2005, 79, 12871-12879. [CrossRef] [PubMed]

36. Someya, K.; Ami, Y.; Nakasone, T.; Izumi, Y.; Matsuo, K.; Horibata, S.; Xin, K.Q.; Yamamoto, H.; Okuda, K.; Yamamoto, N.; et al. Induction of positive cellular and humoral immune responses by a prime-boost vaccine encoded with simian immunodeficiency virus gag/pol. J. Immunol. 2006, 176, 1784-1795. [CrossRef] [PubMed]

37. Kilpelainen, A.; Saubi, N.; Guitart, N.; Olvera, A.; Hanke, T.; Brander, C.; Joseph, J. Recombinant BCG Expressing HTI Prime and Recombinant ChAdOx1 Boost Is Safe and Elicits HIV-1-Specific T-Cell Responses in BALB/c Mice. Vaccines 2019, 7, 78. [CrossRef] 